\title{
Vertical-horizontal illusion: One eye is better than two
}

\author{
WILLIAM PRINZMETAL \\ University of California, Berkeley, California \\ and \\ LAURIE GETTLEMAN \\ University of California, Santa Barbara, California
}

\begin{abstract}
The vertical-horizontal illusion is the tendency for observers to overestimate the length of a vertical line relative to a horizontal line that has the same length. One explanation of this illusion is that the visual field is elongated in the horizontal direction, and that the vertical-horizontal illusion is a kind of framing effect (Künnapas, 1957a, 1957b, 1957c). Since the monocular visual field is less asymmetric than the combined visual field, this theory predicts that the illusion should be reduced with monocular presentation. This prediction was tested in five experiments, in which the vertical-horizontal illusion was examined in a variety of situations-including observers seated upright versus reclined $90^{\circ}$, monocular presentation with the dominant versus the nondominant eye, viewing in the dark versus in the light, and viewing with asymmetrical frames of reference. The illusion was reliably reduced with monocular presentation under conditions that affected the asymmetry of the phenomenal visual field.
\end{abstract}

Two eyes are generally better than one. Tasks in which binocular vision is better than monocular vision include luminance increment detection, contrast sensitivity with sine wave gratings, color discrimination, vernier acuity, letter identification, and visual search, to mention just a few (Banton \& Levi, 1991; Blake, Sloane, \& Fox, 1981; Jones \& Lee, 1981). In the present paper, we report an exception to this general finding. In judging the relative length of vertical and horizontal lines, subjects are more accurate with monocular than with binocular presentation.

One of the simplest visual illusions is the verticalhorizontal illusion. Observers tend to judge a vertical line as being longer than a horizontal line of the same length. In this discussion of the vertical-horizontal illusion, we do not include the form that includes a bisection illusion (Figure 1A) but only the vertical-horizontal illusion in its pure form (i.e., Figure 1B, see Finger \& Spelt, 1947; Künnapas, 1955a). In the experiments reported below, we found that the magnitude of the vertical-horizontal illusion is less with monocular than with binocular viewing.

The notion that the vertical-horizontal illusion would be affected by viewing conditions (i.e., monocular vs. binocular presentation) was motivated by a proposal made by Künnapas (1957a, 1957b, 1957c), who hypothesized

This research was supported by NIMH Grant MH39881 to W.P. We would like to thank Walter Gogel and Irvin Rock for their encouragement. We also are indebted to Irvin Rock, Richard Ivry, and Christopher Linnett for their many helpful comments on the manuscript, and to Beth Silvers for her assistance in data collection. Correspondence concerning this article should be addressed to W. Prinzmetal, Department of Psychology, University of California, Berkeley, CA 94720. that the vertical-horizontal illusion was a kind of framing effect. In general, the closer a line extends toward a surrounding frame, the longer it appears (Künnapas, 1955b). Because the combined visual field (i.e., left and right eyes together) is a horizontally oriented ellipse, vertical lines will generally be closer to the boundary of the visual field than will horizontal lines, and hence vertical lines will appear longer. The combined visual field subtends approximately $200^{\circ}$ versus $130^{\circ}$ along the horizontal and vertical meridians, respectively. Künnapas amassed considerable evidence for his theory. For example, the anisotropy of the visual field is minimized in the dark. Künnapas (1957c) found that the vertical-horizontal illusion was reduced by $32 \%$ when the stimulus consisted of luminous lines presented in the dark (cf. Avery \& Day, 1969). The magnitude of the illusion was also affected by presenting the stimuli in surrounding fields of various shapes (Künnapas, 1957b, 1959). The illusion was reversed when subjects tilted their heads $90^{\circ}$ from the horizontal, making the major axis of the visual field vertical (Avery \& Day, 1969; Künnapas, 1958; Rock, 1975). Finally, when subjects monocularly fixated on the intersection of lines forming an $\mathrm{L}$, the illusion was greater when the horizontal line extended toward the temporal visual field than when it extended toward the nasal visual field (Künnapas, 1957a). This was presumably because when the horizontal extends toward the nasal visual field, it is closer to the visual field boundary.

Künnapas (1957a) predicted that the magnitude of the illusion should be less with monocular viewing than with binocular viewing. This prediction follows from the observation that the monocular visual field is less asymmetric 
than the combined field (i.e., left and right eyes together). Under optimal conditions (i.e., large high-contrast targets), the monocular visual field has a horizontal to vertical aspect ratio of 1.23 , but the combined visual field has an aspect ratio of 1.53 (Harrington, 1981, p. 97). Künnapas (1957a) failed to find any difference in the magnitude of the illusion with monocular and binocular viewing. However, he presented stimuli in a surrounding frame that was elongated in a horizontal direction, thus possibly reducing the effect of the difference between one- and two-eyed views.

Künnapas (1957a) also used a form of the illusion and instructions that might have obscured finding a difference between monocular and binocular presentation. The stimuli consisted of an $\mathrm{L}$ shape, and subjects were asked to fixate on the intersection of the two lines. Künnapas found a large difference in the magnitude of the illusion, depending on whether the horizontal leg of the stimulus extended in a nasal or a temporal direction. This nasaltemporal difference may have concealed a difference between monocular and binocular presentations. In order to avoid this problem, we used a form of the illusion in which the vertical and horizontal lines were not touching and the distance between the lines was great enough to require subjects to separately fixate the lines. This form of the illusion has been used by Begelman and Steinfeld (1967) and by Finger and Spelt (1947).

The main alternative to Künnapas' framing theory is the inappropriate size-scaling hypothesis (Girgus \& Coren, 1975; Gregory, 1963). The inappropriate sizescaling hypothesis is that pictorial depth cues trigger sizescaling mechanisms. A vertical line may be perceived as receding in the distance, and hence it must be longer than a horizontal line that subtends the same visual angle. Whereas Künnapas' theory predicts that the illusion should decrease with monocular viewing, the inappropriate sizescaling hypothesis makes the opposite prediction. This is because the elimination of binocular depth cues should decrease the tendency to perceive the stimulus as two dimensional and should allow the subject to perceive the vertical line as receding in depth, enhancing the illusion. Thus, these two theories of vertical-horizontal illusion can be tested by comparing binocular and monocular stimulus presentation.

\section{EXPERIMENT 1}

In the first experiment, we compared monocular and binocular presentation of two illusions: the verticalhorizontal illusion and the Müller-Lyer illusion. The Müller-Lyer illusion was included to check for general effects of the mode of presentation. The Müller-Lyer illusion does not show anisotropy, and we know of no reason why its magnitude should vary with presentation mode. Hence, we predicted a smaller vertical-horizontal illusion with monocular presentation, but the Müller-Lyer illusion should not be affected.

\section{Method}

Procedure. Each subject was run on four blocks of eight trials. The subjects were tested on the vertical-horizontal illusion on two blocks and on the Brentano version of the Müller-Lyer illusion on two blocks. For each illusion, the subjects were tested on one block each with monocular and binocular vision. The order of the four types of blocks was completely counterbalanced across subjects. For monocular vision trials, the nondominant eye was covered with an eyepatch. The dominant eye was determined by having subjects sight along a ruler as if they were aiming with a rifle. The eye with which a subject chooses to sight is the dominant eye (Porac \& Coren, 1976).

For the vertical-horizontal illusion, on each trial, the subjects were presented with a vertical and a horizontal line in one of the four stimulus configurations that can be generated by reflecting and rotating the stimulus in Figure 1B. For half the trials, the horizontal line was the standard, and the subjects adjusted the length of the vertical line until it matched the length of the horizontal line. On the other half of the trials, the vertical line was the standard. The subjects were told by the experimenter which line to adjust. The order of the eight trials within a block (i.e., four configurations $\times$ vertical/horizontal adjustment) was randomly determined. The subjects adjusted the length of the variable line by pressing one of two keys on the computer's keyboard. Each keypress either increased or decreased the length of the variable line by one pixel. The subjects indicated to the experimenter when they were satisfied that the lines were equal.

A sample stimulus from the Müller-Lyer trials is presented in Figure 2. There were eight versions of this figure, and each version was presented once in a block. Four of the configurations were derived by rotating and reflecting the stimulus in Figure 2, and four other configurations were derived by flipping the direction of the central arrowhead. The order within a block was randomly determined.

The task was to adjust the central arrowhead until it appeared to bisect the line. The subjects responded by pressing one of two keys, and each response moved the central arrowhead one pixel.

Stimuli. The stimuli were presented on a standard Macintosh 13in. $(20.3 \times 25.4 \mathrm{~cm})$ monitor controlled by a Macintosh II computer. ${ }^{1}$ The screen resolution in all experiments was 72 pixels/in. The displays were viewed from approximately $50 \mathrm{~cm}$, although the subjects were free to move their heads. The stimulus lines were white on a dark background and were viewed with normal fluorescent room lighting. The lines were one pixel wide. For the vertical-horizontal illusion trials, the standard line was always 100 pixels long $(3.53 \mathrm{~cm})$ and thus subtended a visual angle of approx-

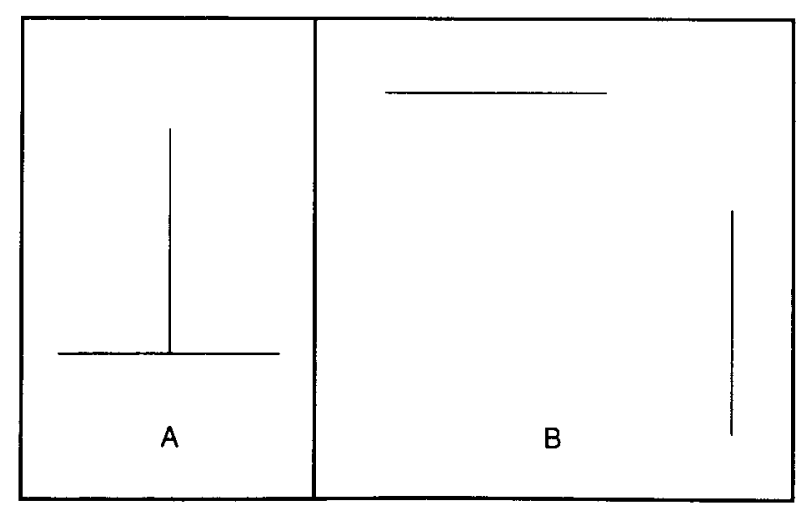

Figure 1. (A) Combination of the vertical-horizontal illusion and bisection illusions; (B) sample vertical-horizontal illusion stimulus. 


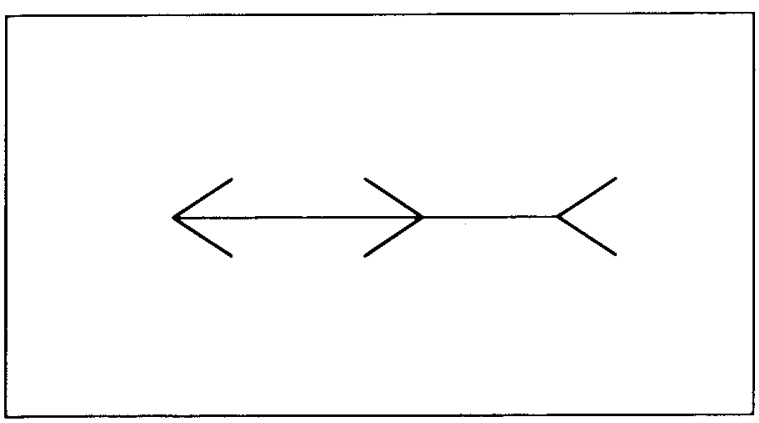

Figure 2. Sample Müller-Lyer stimulus used in Experiment 1.

imately $4.5^{\circ}$. The length of the variable line was randomly determined at the beginning of each trial and varied from 70 to 130 pixels $(2.47$ to $4.59 \mathrm{~cm})$ or a visual angle of approximately $3.2^{\circ}$ to $5.8^{\circ}$. The midpoints of the lines were always separated by $7.48 \mathrm{~cm}\left(9.5^{\circ}\right)$.

For the Müller-Lyer trials, the length of the line was 200 pixels $(7.10 \mathrm{~cm})$ and thus subtended a visual angle of approximately $9^{\circ}$. The length of the arrowhead arms was 36 pixels $(1.30 \mathrm{~cm})$, which is approximately $1.7^{\circ}$ of visual angle. The arrowheads formed a $67^{\circ}$ angle. The location of the central arrowhead was randomly determined at the beginning of each trial and varied \pm 15 pixels $(1.06 \mathrm{~cm})$ from the midpoint of the line.

Subjects. Eight subjects volunteered to participate in the experiment. The subjects were all naive with respect to the purpose of the experiment. The subjects' average age was 26 years; half were female, and all had normal or corrected-to-normal vision.

\section{Results and Discussion}

For the vertical-horizontal stimuli, the magnitude of the illusion was converted to a percentage score. For example, if a horizontal line of 105 pixels was perceived to be equal to a vertical line of 100 pixels, there was a $5 \%$ illusion. The illusion was significantly greater with binocular than with monocular presentation $[F(1,7)=$ $\left.13.20, M S_{\mathrm{e}}=468.9, p<.005\right]$. With binocular presentation, the subjects overestimated the length of the vertical line an average of $2.47 \%$. This is significantly greater than zero $[t(7)=2.47, p<.05$; all $t$ tests reported in this paper tested for an overestimation of the vertical line and thus were one-tailed]. Although the magnitude of the illusion is small, it is in the range found by Künnapas (1955a) and others when the vertical-horizontal illusion has been considered separately from the bisection illusion. With monocular presentation, however, the subjects did not overestimate the length of the vertical line. On the average, they estimated the length of the vertical line to be $1.36 \%$ shorter than the horizontal line, although this percent is not significantly greater than zero $[t(7)=1.9$, $p>$.10]. Thus, if a systematic deviation from the length of a standard is a measure of accuracy, then the subjects were more accurate with one eye than with two. There was no significant effect of the stimulus orientation $\left[F(3,21)=2.52, M S_{\mathrm{e}}=16.17, p>.05\right]$. Thus, by separating the lines, we were able to eliminate nasal-temporal influences.

For the Müller-Lyer stimuli, the dependent variable was the amount of deviation from the midpoint of the line divided by the length of the line. The magnitude of the illusion was $14.80 \%$ and $14.98 \%$ for binocular and monocular presentations, respectively. Both of these numbers are significantly different from zero $(t \mathrm{~s}=21.88$ and 24.74 for binocular and monocular, respectively, $p s<.001)$. However, they are not significantly different from each other $\left[F(1,7)=0.10, M S_{e}=0.95\right]$. Thus, although the mode of presentation affected the verticalhorizontal illusion, it did not affect the Müller-Lyer illusion (see also Porac, 1989). As with the verticalhorizontal illusion, the orientation of the stimulus did not affect performance $\left[F(3,21)=1.61, M S_{c}=16.57\right]$.

In summary, we found that subjects overestimated the length of vertical lines with binocular presentation, but not with monocular presentation. The results from the Müller-Lyer illusion indicate that the difference was not due to some general factor. For example, the effect of presentation on the vertical-horizontal illusion was not the result of subjects' simply being more careful with monocular presentation. The results were consistent with Künnapas's theory, but they were the opposite of the inappropriate size-scaling hypothesis.

\section{EXPERIMENT 2}

In Experiment 1, the subjects viewed the stimuli with the dominant eye. It may be that the reduction in the vertical-horizontal illusion that we observed with monocular vision was due to the fact that binocular presentation involved both nondominant and dominant eyes, whereas monocular presentation involved only the dominant eye. One could account for our results if input from the nondominant eye introduced systematic errors. To test this explanation, in Experiment 2 we tested the verticalhorizontal illusion with binocular and monocular presentation with the nondominant eye.

\section{Method}

To determine eye dominance, the subjects were asked to look into a bottle with a small opening. This test is a variation of the "hole" test, which correlates highly with other measures of sighting dominance (Coren \& Kaplin, 1973). This test, unlike the sighting test, is not confounded with handedness (see Porac \& Coren, 1976). There were four blocks of eight trials. The subjects alternated from monocular to binocular presentation between blocks, with half the subjects beginning with monocular presentation. The verticalhorizontal stimuli and apparatus from Experiment 1 were used. Twelve subjects, with an average age of 25 years, were tested.

\section{Results and Discussion}

As in Experiment 1, the overestimation of the length of vertical lines was larger for binocular than for monocular presentation $[3.56 \%$ vs. $2.39 \%, t(11)=1.90$, $p<.05$ ]. Unlike in Experiment 1, however, the illusion was significantly greater than zero for both binocular and monocular presentation $[t(11)=3.39$ and 3.91 , respectively, both $p s<.01]$. Although monocular presentation did not eliminate the vertical-horizontal illusion, in the present experiment it did significantly reduce it when viewing was restricted to the nondominant eye. 


\section{EXPERIMENT 3}

Research on the vertical-horizontal illusion has been plagued with inconsistencies. For example, Künnapas (1957c) found that the illusion was reduced in the dark, but Avery and Day (1969) failed to find such an effect. Thompson and Schiffman (1974) found that the illusion increased with horizontal eccentricity, but Pearce and Matin (1969) did not. Avery and Day (1969) replicated Künnapas's (1957b, 1958) finding of the effect of a surrounding frame, but only if the comparison stimulus was also within the frame.

We wanted to ensure that there was nothing idiosyncratic about our stimuli or procedure. One finding with the vertical-horizontal illusion that seems robust is that when subjects rotate their heads $90^{\circ}$, the relative overestimation of the vertical line becomes an overestimation of the horizontal line (Avery \& Day, 1969; Künnapas, 1958; Rock, 1975). Thus the vertical-horizontal illusion is in retinal coordinates. This is consistent with Künnapas's frame theory, because when subjects rotate their heads, the anisotropy of the visual field also rotates.

In Experiment 3, we compared the magnitude of the vertical-horizontal illusion with upright and rotated viewing conditions under binocular and monocular presentation. If the overestimation of the length of the vertical line that we obtained in Experiments 1 and 2 is consistent with the illusion that others have obtained, the illusion should rotate with the head. Künnapas's theory predicts that the magnitude of the illusion should be less for monocular presentation in both the upright and the rotated positions.

\section{Method}

The method was identical to that in Experiment 2, except for the following factors. There were four blocks of trials. Two of the blocks were with monocular presentation and two with binocular presentation. For each type of presentation, for one block of trials the subjects were sitting upright in front of the computer, and for the other block, the subjects reclined (on a sofa) with their heads $90^{\circ}$ from the vertical. As before, the order of the four blocks was completely counterbalanced and the order of the eight trials within a block was random. In the monocular conditions, the subjects were allowed to cover either the dominant or the nondominant eye.

The experiment was run on a Macintosh SE computer, with screen dimensions of $18.4 \times 13.7 \mathrm{~cm}$. Unlike in Experiments 1 and 2, the figures were black on a white background. Twelve undergraduate female students at the University of California at Santa Barbara volunteered to participate in the experiment.

\section{Results and Discussion}

In analyzing the results, we coded vertical and horizontal in retinal terms. Overall, the subjects perceived the retinally vertical line as being longer than the retinally horizontal line (see Figure 3 ). This effect was significantly larger in the upright condition than in the rotated condition $\left[F(1,11)=18.48, M S_{\mathrm{e}}=3.26, p<.001\right]$. The average illusion magnitude in the upright and rotated conditions was $7.09 \%$ and $4.85 \%$, respectively. These findings are consistent with Avery and Day (1969), Künnapas (1958), and Rock (personal communication, March 1992),

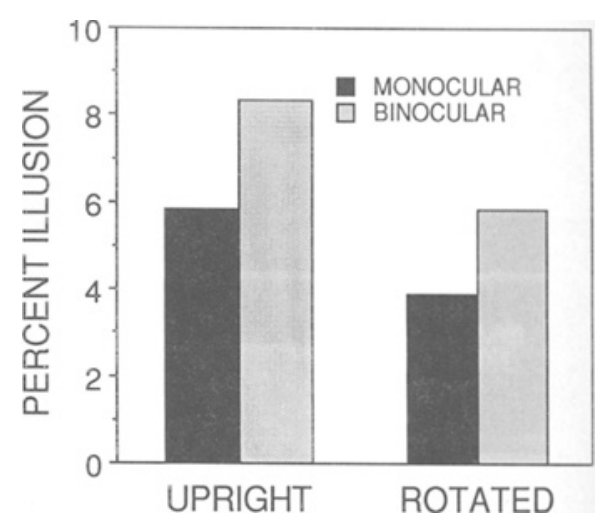

Figure 3. Mean illusion magnitude in Experiment 3. The illusion is the relative overestimation of vertical lines in retinal coordinates.

who also found that although the vertical-horizontal illusion is primarily determined by retinal orientation, the illusion is greatest when retinal and environmental (or gravitational) coordinates coincide.

Importantly, subjects showed a larger horizontalvertical illusion with binocular than with monocular presentation $(7.07 \%$ vs. $4.87 \%)$. This difference was reliable $\left[F(1,11)=150.06, M S_{\mathrm{e}}=0.39, p<.001\right]$. The mode of presentation interacted with head orientation $[F(1,11)=$ 9.63, $\left.M S_{\mathrm{e}}=0.11, p<.05\right]$. The difference between monocular and binocular presentation was greater with the subjects in the upright position than with those rotated $90^{\circ}$, but the difference was significant with both upright and rotated viewing. In the upright viewing condition, the magnitude of the illusion was $8.33 \%$ and $5.84 \%$, for binocular and monocular viewing, respectively $[F(1,11)=167.33, p<.001]$. In the $90^{\circ}$ rotated condition, the magnitude of the illusion was $5.80 \%$ and $3.90 \%$ for binocular and monocular viewing, respectively $[F(1,11)=80.73, p<.001]$. It is not surprising that the reduction in the illusion with monocular presentation was greater with upright viewing, because the overall illusion was greater in this condition.

\section{EXPERIMENT 4}

Experiments 1, 2, and 3 demonstrated that monocular viewing reduced or eliminated the vertical-horizontal illusion. In Experiment 1, the illusion went from $2.47 \%$ to $-1.36 \%$. In the other two experiments, the illusion was reduced by as much as $68 \%$. These results were predicted from Künnapas's framing theory, which attributes the affect of monocular viewing to the fact that the monocular visual field forms a frame that is less anisotropic than the combined visual field. On the basis of this theory, one can predict circumstances in which monocular viewing should have little or no effect on the illusion. In the final two experiments, we examine two of these situations. In Experiment 4, we compared the illusion in a normally lit environment and in the dark. In 
Experiment 5, we examined the effect of the illusion with different surrounding frames.

Künnapas (1957c) hypothesized that the verticalhorizontal illusion should disappear when the stimuli are viewed in the dark because in the dark the visual field does not have a frame. He found partial support for this hypothesis. With binocular viewing, the illusion was significantly reduced from $7.1 \%$ to $4.8 \%$ (Künnapas, $1957 \mathrm{c}$ ). However, Avery and Day (1969) failed to find any reduction in the illusion in the dark. Binocular presentation was used in both of these studies. We cannot resolve the difference between these two findings except by suggesting that Künnapas took greater steps to ensure that there was no visible surround in the dark. Künnapas turned on the room lights between trials so that subjects did not dark adapt, whereas Avery and Day's subjects may have dark adapted. Any stray light might have provided a frame that would have generated the illusion.

The framing theory makes a clear prediction for monocular versus binocular viewing. If the difference between monocular and binocular viewing is due to a framing effect, we should observe it only in the light, not in the dark. This prediction was tested in Experiment 4.

\section{Method}

Procedure. There were four blocks of trials, half with monocular viewing and half with binocular viewing. In addition, in half the blocks, the stimuli were presented in the light, and in half the blocks they were presented in the dark. The order of blocks was fully counterbalanced.

Eight stimuli were presented in a random order in each block: two stimuli for each $90^{\circ}$ rotation of the stimuli similar to those shown in Figure 1B. The room lights were turned on between blocks. Each block of trials took about $2 \mathrm{~min}$. Unlike in the previous experiments, the subjects adjusted only the horizontal line; the vertical line was always the standard.

Stimuli. In Experiments 4 and 5, we used a chinrest both to ensure that the subjects were not surreptitiously tilting their heads in the dark and to control viewing distance. The viewing distance was $95 \mathrm{~cm}$. The standard (vertical) line was always 80 pixels long and subtended a visual angle of $1.67^{\circ}$. The initial length of the horizontal line was randomly chosen to be from 60 pixels $\left(.50^{\circ}\right)$ to 100 pixels $\left(.84^{\circ}\right)$. The stimuli were presented with the same computer equipment that was used in Experiments 1 and 2.

In the monocular condition, the subjects viewed the stimuli with an eye patch over the nondominant eye. Dominance was determined as in Experiment 2. The stimuli consisted of red lines, 2 pixels wide, on a dark background. In the normal lighting condition, the room was lit by overhead fluorescent lights. In this condition, the background brightness of the computer screen was $11.4 \mathrm{~cd} / \mathrm{m}^{2}$, and the brightness of the red lines was $35.5 \mathrm{~cd} / \mathrm{m}^{2}$. The CIE coordinates of the red stimulus lines were $x=.558$ and $y=.362$ (measured with a Minolta Chroma meter, Model CS100).

In the dark condition, the room lights were turned off and the room was made as light tight as possible. However, the dark background of the computer generated a considerable amount of light. In order to attenuate the glow from the computer, the subjects wore spectacles that were fitted with neutral density filters and a Kodak Wratten No. 29 filter (deep red). Phenomenally, only the lines of the monitor were visible with the spectacles. The brightness of the stimulus lines through the filters measured $.24 \mathrm{~cd} / \mathrm{m}^{2}$, and the brightness of the background measured $.01 \mathrm{~cd} / \mathrm{m}^{2}$. Twelve subjects, with an average age of 25 years, were tested.

\section{Results and Discussion}

The percent of vertical-horizontal illusion was subjected to an analysis of variance with the following factors: monocular versus binocular viewing, dark surround versus light surround, and the orientation of the pattern. There was a significant effect of monocular versus binocular viewing $\left[F(1,11)=23.77, M S_{c}=598.55, p<.01\right]$. This effect significantly interacted with lighting condition $\left[F(1,11)=6.04, M S_{e}=178.26, p<.05\right]$. Hence, we separately analyzed the illusion with normal lighting and in the dark.

In normal lighting, there was a significant difference between monocular and binocular viewing $1-.33 \%$ versus $3.09 \%$, respectively, $F(1,11)=36.13, M S_{\mathrm{c}}=1.24$ ), $p<.001$; see Figure 4]. Thus, with monocular viewing, there was a slight tendency to see the horizontal line as longer. The binocular overestimation of the vertical line was significantly greater than zero $[t(11)=2.31$, $p<.05]$. The monocular condition was not significantly different from zero $[t(11)=.32]$.

In the dark, as predicted, the difference between monocular and binocular viewing was not reliable $\left[F(1,11)=1.77, M S_{c}=2.18, p>.20\right]$. The overestimation of the vertical line was $.73 \%$ and $1.73 \%$ for monocular and binocular viewing, respectively. Neither of these numbers was significantly different from zero $[t(11)=.41$ and .98 for monocular and binocular viewing, respectively]. There were no other significant effects or interactions.

The results in this experiment are consistent with the framing explanation for the difference between monocular and binocular presentation. We obtained a difference between monocular and binocular viewing only in normal lighting, not in the dark. Furthermore, we did not obtain a vertical-horizontal illusion in the dark. Several other investigators have obtained a vertical-horizontal illusion in the dark. As discussed above, Avery and Day (1969) explicitly compared lighting conditions and found no difference in illusion magnitude (also see, e.g., Begelman \& Steinfeld, 1967). While conducting this experiment, it occurred to us that it is very difficult to ensure

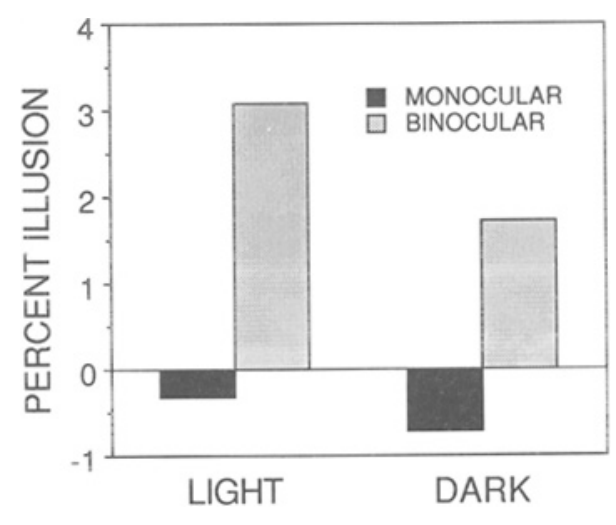

Figure 4. Mean illusion magnitude in Experiment 4. 
absolute darkness and hence the absence of any frame of reference. Any residual light will, of course, have a greater effect when subjects are allowed to dark adapt. Studies in which precautions were taken against dark adaptation resulted in a reduced illusion in the dark (e.g., Künnapas, 1957b; the present experiment), whereas studies in which precautions were not taken against dark adaptation have yielded a vertical-horizontal illusion in the dark (e.g., Avery \& Day, 1969; Begelman \& Steinfeld, 1967).

\section{EXPERIMENT 5}

If the difference between monocular and binocular presentation in the previous experiments was due to the shape of the visual field, the difference should be eliminated by providing an artificial visual field. In the final experiment, we compared monocular and binocular presentation in horizontally and vertically oriented visual fields.

Künnapas (1957b, 1959) compared the extent of the illusion with vertically and horizontally oriented visual fields. He found that the overestimation of the vertical line was enhanced when surrounded by a horizontally oriented frame, and that it was reduced-but not eliminated-by a vertically oriented frame. These results were replicated by Houck, Mefferd, and Greenstein (1972). However, none of these studies explicitly compared monocular and binocular viewing of differently oriented visual fields.

\section{Method}

This experiment was identical to Experiment 4, except for the following. In two of the four blocks of trials, the subjects viewed the stimuli within a horizontally oriented rectangular frame, and in the other two blocks, they viewed the stimuli within a vertically oriented rectangular frame. As in the previous experiment, for two blocks, subjects viewed the stimuli monocularly and for two blocks they viewed the stimuli binocularly.

The frame was created by placing a black matte sheet of posterboard in front of the monitor. Either a horizontal or a vertical opening was cut in the posterboard. The opening measured $16 \times 10 \mathrm{~cm}$. From the viewing distance of $95 \mathrm{~cm}$, the frame created by the aperture subtended $9.6 \times 6.0^{\circ}$ visual angle. The subjects wore the same spectacles as in Experiment 4, but the (red) wratten filters were removed to leave only the neutral density filters. The glow of the monitor was visible through the horizontally or vertically oriented aperture creating the appropriate frame. The room was dark, and only the stimulus lines and the rectangular frame were visible. Through the spectacles, the background light from the monitor measured $.05 \mathrm{~cd} / \mathrm{m}^{2}$, the stimulus lines measured $53 \mathrm{~cd} / \mathrm{m}^{2}$, and the matte black frame measured $.01 \mathrm{~cd} / \mathrm{m}^{2}$.

\section{Results and Discussion}

If the difference between monocular and binocular presentation found in the previous experiments was due to a framing effect, there should be no difference between monocular and binocular presentation when an explicit frame is provided, and that is what we found. For the first time in this research, we found no significant differences between monocular and binocular presentation (see Figure 5). The slightly larger illusion for binocular presen-

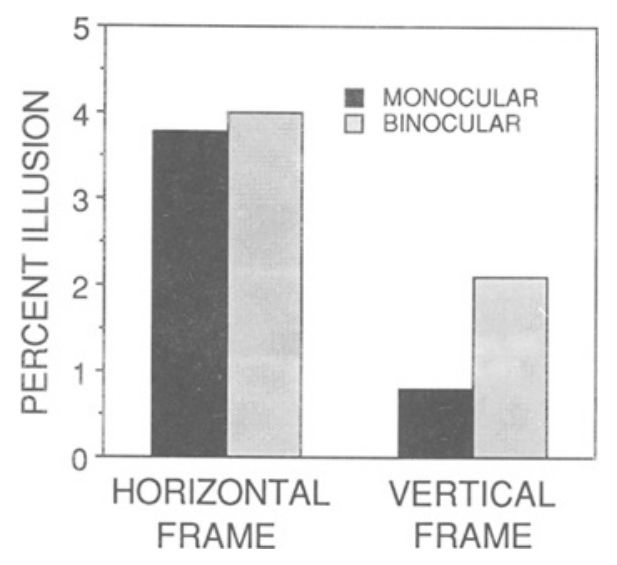

Figure 5. Mean illusion magnitude in Experiment 5.

tation $(3.05 \%$ vs. $2.29 \%)$ did not approach significance $\left[F(1,11)=.75, M S_{\mathrm{e}}=94.73\right]$.

The illusion was larger with a horizontally oriented frame than with a vertically oriented frame $(3.89 \%$ vs. $1.46 \%)$. This difference was highly reliable $[F(1,11)=$ $\left.20.29, M S_{\mathrm{e}}=724.63, p<.001\right]$. The difference between binocular and monocular presentation was larger with the vertical than with the horizontal frame, as is shown in Figure 5, but this interaction was not significant $\left[F(1,11)=.52, M S_{\mathrm{e}}=69.94\right]$. There were no other significant effects or interactions.

In terms of the effect of a frame, we replicated Künnapas's (1957b, 1959) finding that the frame had a large influence in the magnitude of the illusion. Like Künnapas, we also found that the vertically oriented frame did not reverse the illusion. One might expect from the framing hypothesis that a vertically oriented frame should reverse this illusion. However, there may have been a small residual effect of the natural combined asymmetric visual field, owing to a failure to achieve complete darkness. A failure to achieve absolute darkness could explain the small, nonsignificant difference between monocular and binocular presentation in Experiments 4 (dark condition) and in Experiment 5. In the present experiment, the visual field anisotropy and horizontal frame may have combined, making horizontal lines appear shorter. However, in the vertical frame condition, these two influences may have worked against each other to reduce the influence of the vertical frame.

In previous experiments, we may have inadvertently provided a local frame of reference that augmented the vertical-horizontal illusion. The experiment conducted in the light was done with a standard computer monitor that provided a horizontally oriented local frame. This does not invalidate our finding of a difference between monocular and binocular presentation, but the local frame of the computer monitor may have increased the overestimation of the vertical line. Other researchers may also have inadvertently provided a horizontally oriented frame. For example, Schiffman and Thompson (1975; Thompson \& 
Schiffman, 1974) found a vertical-horizontal illusion with monocular presentation. However, they presented their stimuli with a standard tachistoscope that had a horizontally oriented visual field. Thus the vertical-horizontal illusion that they obtained might have been induced or augmented by the surrounding field of the tachistoscope. The use of a horizontally oriented tachistoscope is more problematic than the use of a computer monitor in free viewing because the entire field of view is determined by the frame of the tachistoscope.

\section{GENERAL DISCUSSION}

There is now a considerable body of evidence consistent with Künnapas's theory. When subjects view the stimuli in the dark, the vertical-horizontal illusion disappears, provided subjects are not allowed to dark adapt. Künnapas (1957a) found that the illusion was greater when the horizontal line extended in a temporal rather than a nasal direction. We found that the illusion was reduced or eliminated with monocular viewing. Importantly, however, monocular viewing affected the illusion only when such viewing changed the shape of the visual field. Monocular viewing did not significantly affect the illusion in the dark or when the shape of the visual field was controlled. We confirmed two other observations that are consistent with the framing theory. The illusion was reduced when the stimuli were surrounded by a vertically oriented frame. Finally, the illusion occurred in retinal coordinates.

The inappropriate size-scaling hypothesis cannot account for the present results. There is no reason why pictorial depth cues would be less effective with monocular presentation. On the contrary, one might expect pictorial depth cues to be more important in monocular vision, because the reduction of binocular depth cues would allow the subject to perceive the flat stimulus in depth. Likewise, the inappropriate size-scaling hypothesis would predict that the illusion should be greater in the dark because pictorial depth cues that indicate that the stimulus is two-dimensional are absent in the dark. The size-scaling hypothesis has trouble accounting for other observations. For example, there is no reason why size scaling should operate in retinal coordinates, rather than environmental coordinates. Furthermore, it is unclear that a simple vertical line is a sufficient cue to trigger pictorial depth. Ward, Porac, Coren, and Girgus (1977) found that only 1 in 120 subjects interpreted the vertical line in a verticalhorizontal stimulus as receding in depth. Finally, von Collani (1985a) found that although embedding a vertical-horizontal stimulus in a three-dimensional drawing could enhance the illusion, there was a substantial illusion when the stimulus was part of a figure in which the vertical line was not receding in depth (see also von Collani, 1985b).

Künnapas's framing theory is the strongest current contender for explaining the vertical-horizontal illusion. The framing theory would classify the illusion as a contrast illusion (Gillam, 1980) in which the test elements (i.e., the lines) are contrasted with the visual field and its contents (i.e., the inducing element). ${ }^{2}$ However, quantifying the theory will not be easy for several reasons. First, the extent and asymmetry of the visual field depends on the stimulus that is used to measure the field. The combined visual field measurements given in the introduction $\left(200^{\circ} \times 130^{\circ}\right)$ is for large, high-contrast stimuli. As the stimulus targets that are used to measure the visual field become smaller or lower in contrast, the visual field becomes smaller and less asymmetric (Harrington, 1981, p. 100). Thus the magnitude of the illusion will depend on the size, contrast, and eccentricity of the stimulus. Since the visual field becomes more asymmetric as it increases in size, one might expect the illusion to increase in magnitude as the test elements increase in size. However, the illusion will also depend on the size and contrast of the contents of the visual field. We would predict that the illusion would be reduced with a homogeneous visual field as it was with a dark field. Finally, a particular illusion may show both contrast and assimilation (Coren \& Girgus, 1978, pp. 38-39). As the inducing and test elements become close in size, contrast can change to assimilation. In the Delboeuf illusion, for example, a central circle surrounded by a very large circle will appear smaller than the circle alone (i.e., contrast), but the same circle surrounding a smaller concentric circle appears larger than the circle alone (i.e., assimilation). Higashiyama (1992) measured the perceived visual angle of objects that subtended from $5^{\circ}$ to $80^{\circ}$. As the visual angle to be estimated increased, the relative overestimation of vertical visual angles compared with horizontal angles at first increased, but then decreased.

Although current evidence favors framing as a determinant of the vertical-horizontal illusion, many factors probably contribute to the perceived length of lines. For example, there are findings that present problems for any purely structural account, including framing theory. The observation that the illusion is primarily retinal is consistent with the framing theory. However, the fact that the illusion does not completely reverse when subjects rotate their heads cannot be predicted by any structural account of the vertical-horizontal illusion (see also Ritter, 1917; Thompson \& Schiffman, 1974). Future accounts of the illusion may have to include a cognitive component to add flexibility to structural factors, such as the visual field frame.

Whatever the cause of the vertical-horizontal illusion, the finding of a difference between monocular and binocular presentation has pragmatic implications for researchers interested in the illusion. One might be tempted to study a "pure" vertical-horizontal illusion by using monocular presentation together with either no frame (as in the dark) or an isotropic frame. Our results indicate that such an experiment is likely not to yield any illusion at all. Furthermore, if one wants to maximize the illusion, binocular presentation is best.

It will be interesting to know what other perceptual anisotropies are affected by monocular versus binocular 
presentation. Indeed, there may be other situations where one eye is better than two. We expect that comparing monocular and binocular presentation will be a useful tool in understanding such phenomena.

\section{REFERENCES}

Avery, G. C., \& DAY, R. H. (1969). Basis of the horizontal-vertical illusion. Journal of Experimental Psychology, 81, 376-380.

BAnton, T., \& LEVI, D. M. (1991). Binocular summation in vernier acuity. Journal of the Optical Society of America A, 8, 673-680.

Begelman, D. A., \& Steinfeld, G. (1967). An investigation of several parameters of the horizontal-vertical illusion. Perception \& Psychophysics, 2, 539-543.

Blake, R., Sloane, M., \& Fox, R. (1981). Further developments in binocular summation. Perception \& Psychophysics, 30, 266-276.

Coren, S., \& Girgus, J. S. (1978). Seeing is deceiving: The psychology of visual illusions. Hillsdale, NJ: Erlbaum.

COREN, S., KAPLIN, C. P. (1973). American Journal of Optometry \& Archives of the American Academy of Optometry, 50, 282-292.

Finger, F. W., \& SPELT, D. K. (1947). The illustration of the horizontalvertical illusion. Journal of Experimental Psychology, 37, 243-250.

Gillam, B. (1980). Geometrical illusions. Scientific American, 242, 102-111.

Girgus, J. S., \& CoREN, S. (1975). Depth cues and constancy scaling in the horizontal-vertical illusion: The bisection error. Canadian Journal of Psychology, 29, 59-65.

GREGoRY, R. L. (1963). Distortion of visual space as inappropriate constancy scaling. Nature, 199, 678-680.

HARRINGTON, D. O. (1981). The visual fields: Textbook and atlas of clinical perimetry. St. Louis: C. V. Mosby.

Higashiyama, A. (1992). Anisotropic perception of visual angle: Implications for the horizontal-vertical illusion, overconstancy of size, and the moon illusion. Perception \& Psychophysics, 51, 218-230.

Houck, R. L., MefFerd, R. B., \& Greenstein, G. J. (1972). Influence of a visual frame and vertical-horizontal illusion on shape and size perception. Journal of Experimental Psychology, 96, 273-279.

JONES, R. K., \& LEE, D. N. (1981). Why two eyes are better than one: The two views of binocular vision. Journal of Experimental Psychology: Human Perception \& Performance, 7, 30-40.

KünNaPas, T. M. (1955a). An analysis of the "vertical-horizontal illusion." Journal of Experimental Psychology, 49, 134-140.

KÜNNAPAS, T. M. (1955b). Influence of frame size on apparent length of a line. Journal of Experimental Psychology, 50, 168-170.

KünNaPAS, T. M. (1957a). Interocular differences in the verticalhorizontal illusion. Acta Psychologica, 13, 253-259.
KÜnNAPAS, T. M. (1957b). Vertical-horizontal illusion and the surrounding field. Acta Psychologica, 13, 35-42.

KüNNAPAS, T. M. (1957c). The vertical-horizontal illusion and the visual field. Journal of Experimental Psychology, 53, 405-407.

KüNNAPAS, T. M. (1958). Influence of head inclination on the verticalhorizontal illusion. Journal of Psychology, 46, 179-185.

KünNAPAS, T. M. (1959). The vertical-horizontal illusion in artificial visual fields. Joumal of Psychology, 47, 41-48.

Pearce, D., Matin, L. (1969). Variation of the magnitude of the horizontal-vertical illusion with retinal eccentricity. Perception \& Psychophysics, 6, 241-243.

Porac, C. (1989). Is visual illusion decrement based on selective adaptation? Perception \& Psychophysics, 46, 279-283.

Porac, C., \& COREN, S. (1976). The dominant eye. Psychological Bulletin, 83, 880-897.

Ritter, S. M. (1917). The vertical-horizontal illusion. Psychological Monographs, 23 (4, Whole No. 101).

Rock, I. (1975). An introduction to perception. New York: Macmillan.

SchiffmaN, H. R., \& Thompson, J. G. (1975). The role of figure orientation and apparent depth in the perception of the horizontal-vertical illusion. Perception, 4, 79-83.

Thompson, J. G., \& Schiffman, H. R. (1974). The effect of the magnitude of the horizontal-vertical illusion of horizontal retinal eccentricity. Vision Research, 14, 1463-1465.

voN Collani, G. (1985a). The horizontal-vertical illusion in photographs of concrete scenes with and without depth information. Perceptual \& Motor Skills, 61, 523-531.

von Collani, G. (1985b). Retinal projection or size constancy as determinants of the horizontal-vertical illusion. Perceptual \& Motor Skills, 61, 547-557.

Ward, L. M., Porac, C., Coren, S., \& Girgus, J. S. (1977). The case for misapplied constancy scaling: Depth associations elicited by illusion configurations. American Journal of Psychology, 90, 609-620.

\section{NOTES}

1. The computer programs used to run the experiments reported in this paper can be obtained from the author. They were written in LightSpeed Pascal, and both the source code and compiled versions are available. To receive them, please send a blank 3.25 -in. disk and an appropriate self-addressed stamped envelope to the first author.

2. I am indebted to Irvin Rock for pointing out the relevance to the vertical-horizontal illusion of the assimilation/contrast distinction.

(Manuscript received August 26, 1991; revision accepted for publication June $29,1992$. 